\title{
VP-PET: A New Imaging Modality?
}

$\mathbf{I}_{1}$ field such as nuclear medicine to see an innovation akin to a new imaging modality.

Although in its preliminary stages, the work by Tai et al. (1) on pages 471-479 of this issue of The Journal of Nuclear Medicine describes a novel concept that could be considered as such. Whole-body PET might have enjoyed a dramatic market penetration and growth in the clinical oncology imaging field in recent years (2), but PET overall is by no means new. The tomographic application of annihilation radiation emanating from positrons in its basic form has been with us for more than $32 \mathrm{y}$ (3). Many

\section{See page 471}

significant advances in the technology have occurred in this time, such as the invention of the block detector (4), the advent of whole-body PET (5), fully 3-dimensional data acquisitions (6), fast and dense scintillators (7), the combination of PET with CT (8), and the promising combination of PET with MRI (9). Time-of-flight PET, which has enjoyed a renaissance recently, has also been with us as a concept for a long time (10). Overall though, most of the advantages of PET over singlephoton imaging techniques have been known from the early stages, especially in the lack of the need for a collimator and the resulting high sensitivity and

Received Sep. 19, 2007; revision accepted Sep. 21, 2007.

For correspondence or reprints contact: Arion Chatziioannou, PhD, School of Medicine, UCLA, 700 Westwood Plaza, Box 951770, Los Angeles, CA, 90095-1770.

E-mail: archatziioann@mednet.ucla.edu COPYRIGHT ( 2008 by the Society of Nuclear Medicine, Inc.

DOI: 10.2967/jnumed.107.044222 spatial resolution, with some of the disadvantages, such as increased cost and complexity among others.

The article described here (1) takes a fresh look at the very fundamental geometric implementation of PET and makes use of what others have tried to avoid as an artifact, creating the advantage of a new flexible imaging mode. This mode is introduced as "virtualpinhole PET" (VP-PET) and is based on the fact that a setup with asymmetric detector sizes creates a region with high spatial resolution near the small detector. High-spatial-resolution clinical PET tomographs existed in the past $(11,12)$ but were limited in their capability due to photon noncolinearity (13). This effect, for a symmetric detector setup, limits the spatial resolution of the data to 2.2-mm full width at half maximum for a tomograph with a clinical bore size $(\sim 1 \mathrm{~m})$. In the design proposed here, annihilation photon noncolinearity plays a significantly reduced role, and the result is the possibility for a customized tomographic gantry that can provide very high spatial resolution for localized regions of the human body that are close to the surface. This advance, for the first time, introduces into PET the geometric considerations and the associated magnification advantages of pinhole SPECT. Because of the similarities between this imaging mode and the data acquisitions of pinhole SPECT, the authors coin the technology VPPET. This capability opens up new horizons for implementation in applications that might benefit from high spatial resolution, such as breast cancer imaging and involvement of lymph nodes and the immune system (14). This almost obvious, simple, and elegant geometric solution might be surprising to many who have been working on dedicated PET instruments such as mammography systems (15). The in- creased spatial resolution comes at a cost of two other performance parameters in a similar fashion as for pinhole SPECT — namely, field-of-view (FOV) coverage and photon detection sensitivity. With respect to the latter, the inherent advantages of PET in the lack of a physical collimator, and the possibility to use multiple near high-resolution detectors, could perhaps lead to significantly higher sensitivities than those presented here, although this has not been demonstrated in this work. The use of multiple detectors is a very similar approach to the use of multiple pinholes in SPECT (16). The reduction in the useful FOV might not necessarily be a significant drawback, as the intended use of this imaging modality could be viewed as an adjunct or a magnifying tool for a specific region of interest instead of a replacement of whole-body PET. It is entirely conceivable that after a diagnostic whole-body PET survey, one can determine a location of interest and then focus on the signal emanating from it without the need for additional injection of radiopharmaceutical. With this focused approach, in principle, one can also afford to spend more time in the small region than in any other individual location during the broad PET survey, ameliorating the possible decrease in sensitivity. In this sense, VP-PET is expected to work synergistically with the more traditional whole-body scanners and imaging protocols. Additionally, this technology could find its application in animal imaging. Tomographs with very high sensitivity and spatial resolution are designed with small bores, making imaging of larger animals difficult, if not impossible. The approach presented here could allow imaging of primates, for example, at higher spatial resolution than is possible with a clinical tomograph.

The work described here (1) does not discuss specific implementation issues or possible gantry designs. It 
also does not touch on the issue of whether there is a real need or benefit from fully tomographic acquisition. For example, this imaging mode could certainly be used for "fanbeam" projection imaging of body regions, with the advantages of high-sensitivity coincidence detection, high resolution for near objects, and coverage of regions that dedicated mammography PET systems cannot access. Though if tomographic imaging is desired, it is unclear how one could increase the angular coverage toward a complete tomographic data acquisition, without significantly affecting the detection efficiency between the high- and lowresolution detectors and for covering regions that are not close to the body surface. On the other hand, an increase of the amount of total detector material around the patient, in combination with coincidences within a high-resolution as well as a low-resolution detector gantry, should increase the fraction of the overall detected coincidences, thereby increasing the system sensitivity. This concept of course ignores specific implementation realities such as the need for physically complete photodetectors with associated electronics, adjacent or optically coupled to the scintillator material. Coupling of the high-resolution detectors through optical fibers would not be ideal, as they would introduce additional photon attenuation. Silicon-based solid-state photodetector technology though is approaching practical use, and its application (17) would obviate the need for this complexity. Other issues are clearly in need of additional work in a similar fashion that 3-dimensional PET acquisitions necessitated the development of new image reconstruction algorithms (18). For example, the inclusion of data with nonstandardized spatial sampling and detector efficiency increases the complexity of the image reconstruction algorithm, the normalization process, as well as the scatter radiation fraction-issues that are also not touched in this work. It is expected that if the benefits of this new imaging mode in both spatial resolution and sensitivity are clearly demonstrated, new innovative solutions that will address the implementation will arise.

Despite these shortcomings at this preliminary stage, this work marks the opening of new possibilities in this ever-changing imaging field. Though many aspects of the applicability of this approach to different types of studies will need to be further investigated, this technology seems indeed to be promising.

\section{Arion Chatziioannou School of Medicine, UCLA Los Angeles, California}

\section{REFERENCES}

1. Tai Y-C, Wu H, Pal D, O'Sullivan JA. Virtualpinhole PET. J Nucl Med. 2008;49:471-479.

2. Schoder H, Larson SM, Yeung HW. PET/CT in oncology: integration into clinical management of lymphoma, melanoma, and gastrointestinal malignancies. J Nucl Med. 2004;45(suppl 1):72S-81S.

3. Phelps ME, Hoffman EJ, Mullani NA, TerPogossian MM. Application of annihilation coincidence detection to transaxial reconstruction tomography. J Nucl Med. 1975;16:210-224.

4. Casey ME, Nutt R. A multicrystal 2-dimensional BGO detector system for positron emission tomography. IEEE Trans Nucl Sci. 1986;33:460463

5. Dahlbom M, Hoffman EJ, Hoh CK, et al. Wholebody positron emission tomography. 1. Methods and performance characteristics. J Nucl Med. 1992;33:1191-1199.

6. Cherry SR, Dahlbom M, Hoffman EJ. 3D PET using a conventional multislice tomograph without septa. J Comput Assist Tomogr. 1991;15:655-668.

7. Melcher CL, Schweitzer JS. Cerium-doped lutetium oxyorthosilicate: a fast, efficient new scintillator. IEEE Trans Nucl Sci. 1992;39:502-505.

8. Beyer T, Townsend DW, Brun T, et al. A combined PET/CT scanner for clinical oncology. J Nucl Med. 2000;41:1369-1379.

9. Catana C, Wu Y, Judenhofer MS, et al. Simultaneous acquisition of multislice PET and MR images: initial results with a MR-compatible PET scanner. J Nucl Med. 2006;47:1968-1976.

10. Lewellen TK. Time-of-flight PET. Semin Nucl Med. 1998;28:268-275.

11. Derenzo SE, Huesman RH, Cahoon JL, et al. A positron tomograph with $600 \mathrm{BGO}$ crystals and $2.6 \mathrm{~mm}$ resolution. IEEE Trans Nucl Sci. 1988;35:659-664.

12. Wienhard K, Schmand M, Casey ME, et al. The ECAT HRRT: performance and first clinical application of the new high resolution research tomograph. IEEE Trans Nucl Sci. 2002;49:104-110.

13. Levin CS, Hoffman EJ. Calculation of positron range and its effect on the fundamental limit of positron emission tomography system spatial resolution. Phys Med Biol. 1999;44:781-799.

14. Veit $\mathrm{P}$, Ruehm S, Kuehl $\mathrm{H}$, et al. Lymph node staging with dual-modality PET/CT: enhancing the diagnostic accuracy in oncology. Eur J Radiol. 2006; 58:383-389.

15. Rosen EL, Turkington TG, Soo MS, Baker JA, Coleman RE. Detection of primary breast carcinoma with a dedicated, large-field-of-view FDG PET mammography device: initial experience. Radiology. 2005;234:527-534.

16. Schramm NU, Ebel G, Engeland U, Schurrat T, Behe M, Behr TM. High-resolution SPECT using multipinhole collimation. IEEE Trans Nucl Sci. 2003;50:315-320.

17. Pichler BJ, Judenhofer MS, Catana C, et al. Performance test of an LSO-APD detector in a 7-T MRI scanner for simultaneous PET/MRI. J Nucl Med. 2006;47:639-647.

18. Defrise M, Townsend D, Geissbuhler A. Implementation of 3-dimensional image-reconstruction for multiring positron tomographs. Phys Med Biol. 1990;35:1361-1372. 